\title{
The forgotten coracoid: A case report of a coracoid fracture in a male cyclist
}

\author{
E Duinslaeger, 1,3,4 MD; J H Kirby, 1,2,3 MBChB, MSc (Sports Med); J T Viljoen, 1,2,3 BSc (Physio), MPhil (Exercise Sci); P L Viviers, \\ 1,2,3 MBChB, MMedSc, MSc (Sports Med), FACSM \\ ${ }^{1}$ Institute of Sport and Exercise Medicine, Stellenbosch University, South Africa \\ 2 International Olympic Committee Research Centre, Cape Town, South Africa \\ ${ }^{3}$ Campus Health Service, Stellenbosch University, South Africa \\ ${ }^{4}$ Department of Physical Medicine and Rehabilitation, Catholic University of Leuven, Belgium
}

\section{Corresponding author: P Viviers (plviviers@sun.ac.za)}

\begin{abstract}
A fall onto the shoulder is a common mechanism of injury in cyclists. However, coracoid fractures remain unreported in the literature in this population. These authors report a case of a coracoid fracture missed on the initial plain film radiographs. Whilst these fractures can be easily missed on standard trauma series radiographs of the shoulder, alternate views and other imaging modalities can be used to detect these fractures. Clinical suspicion, judicious imaging and accurate diagnosis of these fractures are important, as stability of the coracoid influences the entire superior shoulder suspensory complex which allows normal function of the shoulder joint
\end{abstract}

Keywords: cycling, trauma, sport, injury, shoulder injury

S Afr J Sports Med 2016; 29:1-2. DOI: 10.17159/2078-516X/2017/v29i0a1636

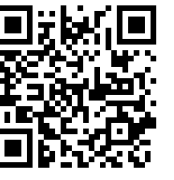

\section{Case report}

A healthy 48-year-old male elite triathlete injured his right shoulder during a descent while mountain-biking. His front wheel hit an obstruction causing him to be catapulted over the handlebars and he landed on his shoulder. He experienced immediate pain, swelling and dysfunction of the shoulder.

On further evaluation there was visible swelling of the superior and anterior aspects of the shoulder without gross deformity. There was diffuse tenderness on palpation, and both active and passive mobilisations were painful. Clinical suspicion was that of an acromioclavicular (AC) joint injury. Patient consent for use of these data in this clinical case presentation was obtained.

Plain film radiographs revealed a cranial subluxation of the distal clavicle on the neutral view, as well as small bony fragments in the proximity of the coracoid but without an identifiable fracture. The $15^{\circ}$ tilt view did not demonstrate a widening of the AC joint (Figure 1A).

Due to the disproportionate pain experienced by the athlete, imaging was followed up with a computed tomography (CT) and magnetic resonance imaging (MRI not shown in this report), using $\mathrm{T} 1, \mathrm{~T} 2$ and fat saturated sequences. The MRI revealed a type II AC injury (according to the Rockwood classification) [1] with oedema of the coracoclavicular ligament and an intact coracoacromial ligament. A fat-fluid level was present in the subacromial bursa and the CT scan demonstrated a splayed fracture of the base of the coracoid process measuring $7.4 \mathrm{~mm}$ with $21^{\circ}$ of angulation (Figure 1B). In addition to the $\mathrm{AC}$ joint subluxation and coracoid fracture, a fracture of the transverse process of the right fifth lumbar vertebra was detected.

Conservative management included the use of paracetamol and a shoulder sling for four weeks with the arm in adduction and the elbow in $90^{\circ}$ of flexion. Following this period of treatment, the patient was still not pain-free and had restricted range of motion. Follow-up CT scans at eight weeks showed callus formation without full union. At 10 weeks post injury, rehabilitation, including shoulder stabilisation exercises and mobilisation, was commenced. Running and cycling were resumed; however, swimming was delayed to protect the AC joint. At one year, the shoulder had full range of motion and activities of daily living were pain-free.

\section{Discussion}

The coracoid process is a key connection between the scapula and the clavicle and an important anchor in the coracoacromial arch; it is part of the superior shoulder suspensory complex (SSSC) as proposed by Goss et al. [2] The SSSC is a ring formed by the glenoid process, the coracoid process, the coracoclavicular ligaments, the distal clavicle, the acromioclavicular joint and the acromial process (Figure 1C). It is strutted superiorly by the middle clavicle and inferiorly by the lateral scapula. A disruption of any one of these structures tends to be a minor injury without compromising of the shoulder complex. Conversely, when the complex is disrupted at two different sites, it becomes anatomically unstable leading to longer healing periods and functional consequences. A double disruption of the SSSC with unacceptable displacement is considered an indication for surgical intervention. [2]

Coracoid fractures are uncommon, and account for $1 \%$ of all fractures and $3-13 \%$ of scapula fractures in the general population. These fractures have not been previously reported in cycling. The precipitating event is typically a 


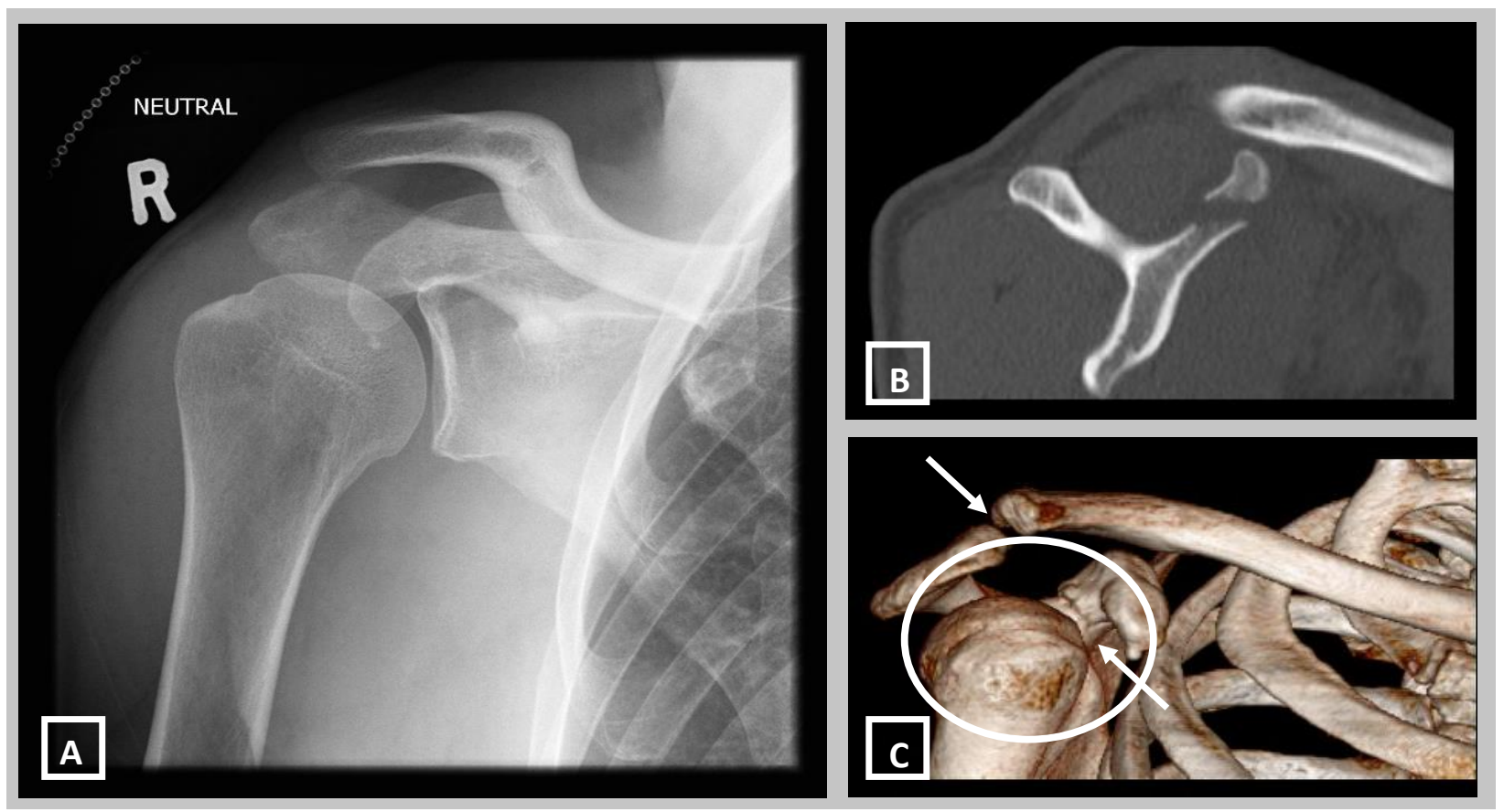

Fig. 1A. Anteroposterior X-ray view of right shoulder shows a cranial subluxation of distal clavicle without identifiable fracture.

Fig. 1B. Computed tomography scan showing a basal fracture of the coracoid process.

Fig. 1C. 3D CT images of SSSC ring (circle) with AC dislocation and basal coracoid fracture (arrows).

direct blow to the lateral shoulder (being thrown over the handlebars); however, this mechanism more commonly causes AC joint injuries and clavicle fractures; which are reported as the most frequent traumatic injuries in cycling.[3] The possible mechanisms leading to the fracture are coracoclavicular ligament avulsion during an AC dislocation; a direct blow to the process, or extremely forceful contraction of the biceps and/or the pectoralis minor muscles. Associated injuries are among others acromial fractures, glenohumeral dislocations, clavicle fractures and AC joint dislocations. [4] According to Haapamaki et al. the diagnosis of coracoid injuries is frequently missed; [5] however, a lack of organised epidemiological reporting of injuries within the cycling fraternity may contribute to this observation.

Plain film radiography is the first-line imaging modality; however, the coracoid process is difficult to visualise with routine views due to the superimposed structures. The sensitivity of primary radiography (AP-view, scapular $\mathrm{Y}$ and axial projection) in the diagnosis of coracoid fractures is $40 \%$, making these fractures the most occult of the scapula fractures. Moreover, due to associated injuries, the focus of attention is drawn away from the coracoid. [5] Likewise, in this case the coracoid base fracture was missed on initial radiographs. To improve diagnostic accuracy, the Stryker Notch View is recommended as the scapular $\mathrm{Y}$ view sensitivity is poor. [5]

The significance of making an accurate diagnosis of a coracoid fracture would allow the clinician to assess the stability of the shoulder complex, and to institute the correct treatment plan bearing in mind the associated injuries. This would in turn facilitate a quicker return to functionality without secondary consequences, such as nonunion, malunion, coracohumeral impingement or shoulder instability. $[2,6]$

In conclusion, a typical fall onto the shoulder could result in a coracoid fracture. These fractures are frequently overlooked on standard X-ray views but can be more easily demonstrated in a cephalad-tilted view or an adapted Stryker Notch View, which is useful in this setting. With this case the authors would like to encourage clinicians and radiologists to actively exclude coracoid fractures with appropriate careful examination and specific radiological views. The shoulder consists of an intricate suspensory complex and therefore the complete assessment of all these structures is important to formulate an accurate diagnosis and appropriate treatment plan in order to avoid delayed healing and further negative functional consequences.

Acknowledgements: The authors would like to thank Winelands Radiology for the images.

\section{References}

1. Rockwood CA, Matsen FA,Wirth MA,et al. The shoulder. Vol.1. 4th ed. Philadelphia: WB Saunders, 1990:533-537.

2. Goss TP. Double disruptions of the superior shoulder suspensory complex. J Orthop Trauma 1993;7(2):99-106. [http://dx.doi.org/10.1097/00005131-199304000-00001] PMID:8459301

3. Silberman MR. Bicycling injuries. Curr Sports Med Rep2013 SepOct;12(5):337-345. [http://dx.doi.org/10.1249/JSR.0b013e3182a4bab7] PMID:24030309

4. Ogawa K, Yoshida A, Takahashi M,et al. Fractures of the coracoid process. J Bone Joint Surg Br 1997 Jan;79(1):17-19. [http://dx.doi.org/10.1302/0301620X.79B1.6912] PMID:9020438

5. Haapamaki VV, Kiuru MJ, Koskinen SK. Multidetector CT in shoulder fractures. Emerg Radiol 2004 Dec;11(2):89-94 [http://dx.doi.org/10.1007/s10140-004-0376-x] PMID:155174535.

6. Gerber C, Terrier F, Ganz R. The role of the coracoid process in the chronic impingement syndrome. J Bone Joint Surg Br 1985 Nov;67(5):703-708. PMID:40558642011;127(3):511-528. [http://dx.doi.org/ 10.1542/peds.20093592] 